\title{
Ethical Reflections on the Conduct of HIV Research with Community Members: A Case Study
}

Journal of Empirical Research on Human Research Ethics 202I, Vol. I6(I-2) 65-77 DO!. 10.1177/1556264620970802

journals.sagepub.com/home/jre

\author{
Brandon Brown', Jeff Taylor², Karine Dubé3, Dario Kuzmanović1,4, \\ Yasmeen Long ${ }^{5}$, and Logan Marg'
}

\begin{abstract}
Some researchers continue to engage in "helicopter" or "parachute" research and do not ethically engage or collaborate with communities from which data are collected. This paper uses a case study to discuss the ethical issues arising from these research practices and the importance of increasing meaningful community involvement in research. Set in the context of research among older people living with HIV, the case study is followed by the perspectives of four research stakeholders. Through these perspectives, this paper demonstrates the ethical perils and harms that stem from research practices exemplified in the case. We argue instead for researchers to practice participatory research methods in line with community-based participatory research approaches (CBPR), good participatory practices (GPP), the Denver Principles, and CIOMS guidelines. Towards this end, we describe tools developed in collaboration with stakeholders in the research process to help researchers incorporate community participation and reduce unethical research conduct.
\end{abstract}

\section{Keywords}

Ethics, lessons learned, HIVIAIDS, community, participatory research

\section{Introduction}

While the HIV and AIDS research field has a long history of fostering relationships and partnerships among community representatives, community-based organizations (CBO), and academic researchers, some researchers struggle with thoughtfully and meaningfully engaging and collaborating with the communities from which they collect data (Rhodes et al., 2010). As a result, some researchers engage in research practices described as "helicopter" or "parachute research," in which they drop into communities, gather data or specimens, and leave (cynically, these studies are sometimes called "bleed and flee" research) (Smith, 2018). In such cases, the research may be primarily in the interest of private companies or researchers, rather than improving the health, quality of life, and social equity of the community from which the data were gathered (Brown \& Sugarman, 2020; Subica \& Brown, 2020). Further, community members are often left with little information about the study and/or study outcomes (Bastida et al., 2010). Such parachute research practices can create mistrust between disadvantaged communities and researchers, as well as harm researchers' credibility, the reputation of the associated research institution, and future opportunities for research that could actually benefit disenfranchised populations and communities (Bastida et al., 2010). This cascade can reduce willingness to participate in research, and undermine trust in biomedical science. It also falls below recommended standards for participant research (Barrett et al., 2020; Browne et al., 2019).

While community engagement is often deemed an essential component of ethical research, it does not always occur in research practice (Brown \& Sugarman, 2020; Emanuel et al., 2004, 2008). Community-based participatory research approaches (CBPR) emphasize a more equitable partnership between researchers and community members throughout the entire research process, in which community members are treated as true partners and experts in the health and social problems that affect their lives (Rhodes et al., 2010). By involving community members as partners

\footnotetext{
'University of California, Riverside, CA, USA

${ }^{2}$ HIV \& Aging Research Project-Palm Springs, Palm Springs, CA, USA

${ }^{3}$ University of North Carolina, Chapel Hill, NC, USA

${ }^{4}$ The Joint Centre for Bioethics, University of Toronto, Canada

${ }^{5}$ FasterCures at the Milken Institute, Washington, DC, USA

Corresponding Author:

Brandon Brown, University of California, 900 University Ave, Riverside, CA 92521, USA.

Email: brandon.brown@medsch.ucr.edu
} 
in the research process, researchers and community members learn together and from each other, and aim for shared decision-making (Rhodes et al., 2010; Wallerstein \& Duran, 2010). This type of partnership also strives to equalize some of the inherent power differences, build trust, improve understanding and voluntariness of consent, and foster mutual ownership over the research and its outcomes (Adhikari et al., 2019; Rhodes et al., 2010; Wallerstein \& Duran, 2010). Research using CBPR is often better aligned with the priorities and needs of the population under study, which can improve the translation of research findings into interventions and other actions to enhance health, quality of life, and social equity (Wallersteinet al., 2018). Given the myriad benefits of CBPR and increasing demands that research better reflects communities' needs, perceptions, and circumstances, health disparities research-including HIV research-is increasingly conducted using CBPR (Wallerstein et al., 2018).

The purpose of this paper is to utilize a case study to help researchers, IRB members, community members, and other stakeholders understand the importance of enacting researcher-community partnerships throughout the entire research process for all research, and following best-practices for doing so ethically and meaningfully. While the case study was not conceived as CBPR in the mind of the researcher, the community lead had come to expect a CBPR approach due to their history of participatory collaborations in research.

\section{Methods}

The case study was developed organically and is based on events that occurred. The community lead developed the case study based on their experience with the researcher, and discussed it with the corresponding author. Together they worked to present the case as objectively as possible. Once the case study was finalized, we collected perspectives about the case study from four key research stakeholders via email outreach. Stakeholders were all known to the corresponding author, all involved in research, and except for the community member, were fully independent of the case study presented here. The stakeholders include a community member, socio-behavioral researcher, an institutional research board (IRB) representative, and a program officer from a non-profit funding organization. Stakeholders were provided with the following questions along with the case study: (1) what do you think about what happened, (2) what are the negative/bad/violations that happened if any, (3) what are the positive/good/constructive things that happened if any, (4) what should have happened, (5) what people in your role can do to correct this, (6) how do we prevent any negative/bad/violations (if any) from happening in the future, (7) what would you say to the researcher (at the beginning, middle, or end of the scenario), and (8) what would you say to the local community leader or the community itself (at the beginning, middle, or end of the scenario). This case study was exempt from IRB review as it does not meet the definition of "human subjects research." No private identifiable data were collected and the focus was on subjective data collected from stakeholders for nonresearch purposes.

Each stakeholder first independently provided their perspective, and then all responses were circulated among stakeholders for an opportunity to further revise their perspectives. Next, each stakeholder independently extracted cross-cutting themes and sent them to the corresponding author, who circulated the themes amongst all stakeholders. Then stakeholders evaluated the cross-cutting themes and reached consensus on the key themes to be elaborated upon in the discussion. Through these perspectives about the case study, this paper sets out to describe perceived pitfalls on the part of the researcher and community leaders. Following the perspectives, we discuss the core components of the stakeholders' perspectives and their alignment with ethical principles and practices for conducting sound, communityengaged research. We discuss the case study in terms of norms from CBPR and GPP. Finally, we describe tools we developed in collaboration with stakeholders in the research process to help assure better and more meaningful community participation and significantly strengthen ethical conduct in future research.

\section{Case Study}

The following case study is an anonymized account of events that transpired between an HIV researcher and local community members, including the community stakeholder, whose perspective is included in this paper. The text appears exactly as it was presented to the case reviewers via e-mail, with minimal identifiable information removed as necessary to maintain confidentiality of key actors. The case study was not planned as a CBPR project.

The HIV researcher, "Dr. JM," [identifying information removed] works out of state and asked a local community leader in [identifying information removed] to collaborate on a survey on HIV and aging. The community leader agreed to collaborate to advance knowledge on this important topic. Dr. JM said they wanted this survey, which originated in the context of an existing cohort, to be used in "a real-world community setting." The researcher did not share pertinent study-related materials (informed consent form, survey) with the local community prior to IRB submission. When asked to share a draft of the informed consent form and survey with the local community advisory board, the researcher refused to do so. The researcher would yell at the community leader over the phone for 
making what the researcher perceived to be unreasonable requests, including sharing study-related materials. Once the application was approved at Dr. JM's institution, the researcher was adamant that written IRB approval was required to share materials with anyone not listed in the application. The community leader was asked to find community volunteers to administer the survey to their peers and qualified people were identified to do so. In order to administer the survey, the community volunteers completed their online Collaborative Institutional Training Initiative (CITI) research ethics training certificate which took approximately 3 hours for each person. These community volunteers asked again to see the survey prior to the day of administration to better understand the study, but Dr. $\mathrm{JM}$ refused to share the project survey until the date of recruitment. The community leader asked repeatedly to see the survey and informed consent form. In response, the researcher threatened to cancel the project. The community leader and his non-profit had invested considerable funds to rent space at a community event to conduct the survey, which would have been lost if the researcher backed out at that time. Once the informed consent form was shared, the community leader and community volunteers discovered several problematic issues. For example, they learned that the survey was exclusively aimed at men who have sex with men (MSM) and transgender women, excluding cisgender women and transgender men. In Dr. JM's survey, transgender women were considered to be MSM, and the outdated and stigmatizing language of "HIV infected" was used in the study rather than less stigmatizing terminology of "people living with HIV." Community volunteers also learned that no incentives would be provided to study participants, which they would have known if allowed to review the protocol ahead of time. They also learned that there was no plan in place to disseminate the results to the participant community, or to share data with the community leader.

\section{Results: Short Perspectives}

The following are the individual independent perspectives provided by each of the four stakeholder groups, including the community member, socio-behavioral researcher, IRB representative, and the program officer.

\section{Perspective from Community Member: [identifying information removed]}

This is a classic example of "helicopter research," in which an outside academic researcher enters a community to conduct research to further their own research [career], rather than benefit the community with whom they are partnering. In this situation, it appears the researcher's goal was to validate the findings from [an earlier research study] by cherry-picking participants from the community to exactly match the participants in the previous cohort. Proving reproducibility of results with an identical study population does not really teach us anything new and is a misuse of willing community participants and collaborators. Certainly, in this situation there would have been plenty of participants to compare to those in the original setting to prove the reproducibility of the original cohort study, while at the same time providing their community collaborators with valuable data on everyone in their community. Doing so would have benefited both the academic and community research partners.

Community-based research is done in the "real world" and is therefore messier than research conducted in more controlled settings. Academic researchers would be wellserved to view it as translational research, in which results obtained in carefully controlled research settings are then tested in a real world community with much more heterogeneity in participants, the environment, and other variables. This is the best way to test whether the findings from controlled settings are applicable in the real world. If not, no matter how rigorously that research may have been conducted in a controlled environment, those findings have no applicability or utility in the real world. Many cohorts were designed decades ago, and no longer reflect the current diversity of the affected community. One good example is people from the transgender community, who were once lumped together with gay men. Not only can this confound the data collected by including disparate populations in the same group, but by ignoring and dismissing their gender identity, it devalues them as people. Community research collaborators know their own communities and can assist academic researchers in designing studies that address those realities to provide better and more usable results.

Obviously, communication between the academic researcher and community collaborator in this situation was suboptimal. Better communication could have prevented this situation and made it a more productive and trust-building collaboration.

Here are some other lessons that can be learned from this scenario. Both parties (i.e., researchers and community partners) should complete a memorandum of understanding (MOU) clearly outlining all aspects of the collaboration, including: study populations, study objectives and aims, participant/stakeholder review of the entire study prior to IRB submission to ensure the study is designed and conducted in a culturally competent manner (including the use of people-first language), compensation for all study volunteers, compensation for community volunteers who assist in the research, thank you letters to study volunteers at end of study, a dissemination plan that involves reporting study results to the community, and acknowledgement of study participants and the community in all presentations and publications of the study results. Additionally, any required trainings (e.g., CITI, study participants protection, etc.) 
for community volunteers conducting the study should be provided in user-friendly formats - not necessarily the formats used at institutions designed for academic researchers.

Conducting research with, not in, a community is an invaluable opportunity to test hypotheses in a current real world setting free of past biases that can affect the validity and relevance of results obtained from cohorts created decades earlier. Situations like this are problematic in that they can erode the community's trust not just in certain researchers, but in the entire research process. Past experiences like Tuskegee and Henrietta Lacks still resonate strongly in many communities today-limiting the ability to perform much needed research that could benefit those communities.

Researchers should view the community-including potential research participants and research collaboratorsas a resource to partner with, not to exploit. Well designed and executed academic and community research partnerships can provide both the academic and community partners with data that not only furthers science, but also benefits the participants and their communities who make this research possible.

\section{Perspective from Socio-behavioral Researcher:[identifying information removed]}

In reading through the scenario, the first thought that came to mind was that this is an example of self-serving parachute science. The approach did not strike me as collaborative or transparent. The HIV researcher wanted to gain access to the population of interest, instead of serving the needs of the community. Further, this case seems to have violated the three Belmont Report principles (Department of Health, Education, and Welfare, 2014). First, representational justice - defined as the fair distribution of the costs and benefits to potential study participants - was violated since the survey excluded key populations of interest (cisgender women and transgender men). Broad representation of aging PLWH could have ensured more equitable access to the research. Second, respect for persons and communities was violated, since community members were not treated as equal partners. Transgender women were counted as MSM, disrespecting gender identities. Third, in the name of beneficence, benefits of the research were not maximized since the HIV researcher failed to share the findings with the community. One can also wonder whether exploitation took place, since benefits and burdens appeared to have been unfairly distributed from the research transaction.

The case study explains the various faux pas that occurred. The omission to share study documents and obtain community input was an important one. Community members were used as "data collectors," and the research project was imposed on them from the outside. The HIV researcher also used stigmatizing language in study documents (people-first language is preferred). While the lack of compensation for study participants is not a violation-as long as it was approved by the IRB - the lack of process and community consultation around how incentives were defined undermined the ethicality of the research. The project did not have a data sharing or dissemination plan and it appears the community could have benefited from having access to the findings.

I found two positives from the case: (1) the community leader wanted to advance the research and collaborated to ensure the research took place, and (2) community members were offered additional training, strengthening their capacity.

When working with communities, it is important to clarify expectations from the outset, and to ensure mutual understanding and dialogue. The process should have been more inclusive, providing community members with mechanisms to provide input into the research process, including reviewing study documents and helping determine research priorities and questions. Important study documents should have been shared and prepared in an iterative process. I am not aware of any IRB requirement around sharing or not sharing of study materials with key stakeholders. If there were concerns around keeping documents private, a confidentiality agreement could have been signed by all parties involved. The team should also have a data sharing plan, and key findings should have been shared with community members in lay terms after the study - even prior to publication of the data. The data dissemination step is often skipped over in the research process; yet, it is a critical opportunity to draw lessons learned and prepare for the next research cycle. The HIV researcher could have involved community members in interpretation of the data, and even offered co-authorship or due acknowledgements to them. Ensuring community voice is also an important element of a data dissemination strategy, since it increases community autonomy and allows meaningful community dialogue to occur.

I firmly believe that community members should have been treated as equals and true partners in the research process. The community leader should perhaps have been a coinvestigator on the study protocol. In cases like this one, the process is much more important than the final research outputs. Preserving the long-term trust and the partnership with the community is more important than producing peer reviewed publications. The research process could have been a unique opportunity to solidify community links and build support for current and future studies. Researchers must observe GPP, including respect, mutual understanding, integrity, transparency, accountability and community/ stakeholder autonomy. While ensuring due process takes more time, it builds trust and results in better collaborations 
in the long-term. The researcher should have also been willing to leave something behind and to make the community better off than before the research started.

Perhaps there should have been a MOU-elaborated either formally or informally-to determine the type of community and patient involvement needed to ensure the success of the project. The researcher should have also asked the community members how they wanted to be engaged, and what would be most valuable to them in terms of research. The researcher should have made community members feel valued and comfortable giving input, listened to community concerns, and followed recommendations. Promoting active engagement and dialogue throughout the entire research process would have reduced the risk of tokenistic involvement.

I would have asked the researcher to put themself in the community leader's shoes, and truly consider how the research could benefit the community. The researcher should have also tried to work towards aligning their interests with the interests of the community. They should understand that the lack of a deliberative process is a real issue that has negative consequences beyond one's own research, since it builds distrust in academic research. They should ask what true community-based participatory research looks like, and even take a course on the subject before embarking on the research. They should also pay attention to language choices, as this can go a long way in building trust and ensuring the research is culturally sensitive. They should also spend some time reading key sources of guidance for conducting ethical research with community members (MacQueen et al., 2012; Partner, n.d.; Shippee et al., 2015; "The Denver Principles," 1983; UNAIDS/ AVAC, 2011).

I would have reminded community members that they are in the driving seat, and not passengers on the bus. Community members have the right to self-determination and to be acknowledged and treated as equal in the research process. Community members should also sometimes remind researchers that participating in research has a cost and that their time is valuable. In fact, there is oftentimes a problem with community engagement being perceived as "voluntarism" or "service," when it should be built as part of the research process and properly compensated for (this is work!). Ultimately, community members have the right to veto the research, decide to withdraw from the project, and exercise their right of voluntariness. Community-engaged research should be just that - community-engaged.

Ultimately, as researchers, we need to pay attention to all "intangibles" involved in the research process. We need to be mindful of how we can make people feel. While this can sometimes be considered the "soft side" of research, it is extremely important, since research is also about building relationships. We need to be careful that we are not making the community feel like the research is taking something away, but that we are giving back to the community for all that it contributes. I also believe researchers should be asked to pass a test in community/stakeholder engagement and cultural awareness, and that this should even be required by funders, along with IRB approval (AVAC, 2018). The disability rights movement slogan "Nothing About Us Without Us" is a call for social justice but also applies in all research situations and should serve as the foundation for how we do research.

\section{Perspective from IRB Representative: [identifying information removed]}

The case presents an interesting dilemma on what qualifies as ethical research and what is research that follows the minimal regulatory standards of compliance. While the case could easily be seen as unethical research that has failed to adequately engage the local community partners, from a regulatory or IRB perspective, there is very little that can be done. The U.S. regulations governing research with living individuals, the so-called "Common Rule," strictly speaking only applies to studies with federal funding (U.S. Department of Health and Human Services, 2017). Many institutions that receive federal monies, such as colleges and universities, choose to apply the Common Rule more broadly to all studies, such as non-federally funded ones. Even assuming that this study is federally supported, very limited recourse is available to the community leaders who may feel disrespected.

The U.S. Common Rule provides a minimal standard of research compliance and was largely animated by egregious research violations in the second part of the 20th century (e.g., U.S. Public Health Service-funded Tuskegee experiments, Milgram experiments, 1966 Beecher article, etc.) (Beecher, 1966; Brandt, 1978; Milgram, 1963). The Rule does not say anything about study team dynamics nor takes any definitive, let alone prescriptive, stands on community engagement. As a matter of fact, the only relevant time the word "community" is mentioned in the Common Rule is in 45 CFR 46.107 (a) under the section IRB membership:

\footnotetext{
"The IRB shall be sufficiently qualified through the experience and expertise of its members, and the diversity of the members, including consideration of race, gender, and cultural background and sensitives such as community attitudes, to promote the respect for its advice and counsel in safeguarding the rights and welfare of human subjects" (U.S. Department of Health and Human Services, 2017).
}

Most institutions meet this membership requirement by having one or two community members on the Board. Considering the variety of research that an average IRB could see in any given meeting, these individuals cannot be expected to represent the attitudes of all possible research 
participants and communities. Given the study methodology and nature of survey research, this study was most likely reviewed via the delegated review process, which means that one member of the IRB reviewed it on behalf of the Board - most certainly not a community member.

Dr. JM's claim that written IRB approval was required to share materials with anyone not listed on the application does not seem to be supported by any identified ethics guidance. Barring any unusual institutional requirements, IRBs usually assume that researchers will share their study materials with those who have a legitimate need to see them-especially community advisory boards and other collaborators. If the community leaders were to bring a formal complaint to the researcher's IRB noting all the grievances from the case, the Board would most likely dismiss it since IRBs usually only consider complaints from research participants and not community collaborators. Most IRBs would view this type of issue as inter-study team conflict and outside of their scope of responsibility. The Board's dismissal should not be surprising given the chorus of criticisms from researchers against IRB oversight and "ethics creep." Ethics creep has been defined as expanding the reach of ethical oversight beyond that which is contemplated in the regulations, such as the Common Rule (Haggerty, 2004). From an IRB perspective, the person responsible for the conduct of the study is always the principal investigator (PI). Given that Dr. JM is a professor at a university, nothing could be done to remedy the situation from the IRB's perspective since the PI decides who gets to see the study documents and when (if at all) the sharing of those documents should happen.

It bears keeping in mind that the community collaborators always had a choice not to take part in the study. The loss of the deposit notwithstanding, the community partners were not coerced into participating in this research project by the researcher (Klitzman, 2013). Most of the negative issues that come out of this case seem to be due to a lack of communication and difference in expectations. At the outset, clear expectations of roles should have been outlined and communicated between all parties on the project. A generous interpretation of events in the case might imply that the researcher saw the individuals from the community to be more along the lines of study coordinators who are recruited to help execute the study; on the other hand, the community members might have seen themselves as study advisors or collaborators who were meaningfully engaged in the research process and knowledge creation. The difference in these two roles is stark and not easily reconcilable.

The former role is more transactional, hierarchical, and in line with what seems to have transpired in this case. However, this type of a role usually carries some form of compensation for the community members who are asked to perform a service on behalf of the study PI. The latter role would require more consultation and collaboration with community associates. It would certainly result in a survey that is more sensitive to local needs. HIV and AIDS research has a long tradition of CBPR. However, CBPR is not practiced universally with all researchers who work on HIV and AIDS research. Some HIV researchers use CBPR approaches exclusively, while others use them selectively or not at all. A survey that meaningfully engages the community during creation and execution would also generate more valid data that is generalizable and has real world implications, which was the researcher's stated intent. Needless to say, it would also show respect for members of the transgender community and not expose participants to antiquated and stigmatizing language during data collection.

While not violating any regulatory requirements, such as those outlined in the Common Rule, we argue that this study had several ethical shortcomings, as argued by our interviewees. Most people would not consider the study to be ethical research. One positive outcome might be that this would be a learning experience for the community leaders who may now be more selective about which researchers they partner with and will be more diligent in setting expectations and procedures for key deliverables (such as drafting survey questions, reviewing the IRB application, providing input on study design and dissemination of results, etc.). Additionally, the researcher might also learn to be more explicit in what they expect from community leaders and what they see their role in the study to be. Candid and difficult conversations about expectations and roles must take place as part of project planning.

Many in the research enterprise look to IRBs as arbiters to find solutions to the thorniest ethical issues that may arise during the conduct of a study. But IRBs are only responsible for a small subset of contentious issues focused mostly on participant safety. The world of research is full of ethical issues; however, not all of them can be the responsibility of the IRB.

\section{Perspective from Program Officer: [identifying information removed]}

This case study is a prime example of how communities and populations considered to be vulnerable are not valued and respected for the knowledge and expertise they are capable of bringing to the research. For instance, in this case, the researcher was not respectful because the survey volunteers and study participants were not valued for their time commitment or efforts. The researcher's refusal to share the survey instruments with the volunteers undermines the quality of the data collection and its results and shows a lack of respect for the community. If the volunteers are not permitted to review the survey prior to administering it to study participants, how can the volunteers inform the participants about the purpose of the 
research? What benefit will the research have on the aging community living with HIV? It is the responsibility of the researcher to treat community leaders with respect while maintaining research integrity. Community-based organizations (CBOs) and community advisory boards are a rich resource for researchers conducting health research in communities of interest.

The inclusion of the community supports the development of IRB study materials and the active recruitment of participants. These activities can be beneficial if used as a mechanism for creating partnerships to minimize power differences between researchers and community leaders. When CBOs and community leaders are engaged in the development and review of the research materials, they are in a better position to advise on the study design. This could assist with eliminating unethical treatment and disrespect. There is a large literature on stakeholder engagement (CTSA, 2011; HANC, 2014; MacQueen et al., 2012; The Denver Principles, 1983; UNAIDS/AVAC, 2011).

The detached and depersonalizing treatment of community members poses many risks, such as mistrust of the researchers, misuse of time and resources, and/or low-quality research outcomes. The behavior of the researcher was experienced by some as dismissive, manipulative, and unethical because it included withholding key information. In this case, the community leader should have determined whether the research should continue and if the risks outweighed the benefits.

The researcher should have established preliminary meetings with community leaders. Specifically, the researcher and community leaders should collaborate on adapting existing research materials to ensure they are aligned with the needs and priorities for this community of PLWH. A purpose of engaging with community leaders and study volunteers is to maintain positive relationships with researchers. One strategy the researcher could use is to invite the community leaders to serve as consultants during the development of study materials to ensure they were applicable to this community. Also, community volunteers and study participants should have received compensation or other incentives for their time because this demonstrates that the community is valued.

There are methods that can be used to meaningfully involve communities. A best practice includes inviting community leaders to partner with the researchers as stakeholders (Shippee et al., 2015). For example, community leaders can advise researchers on the appropriate language to support the successful recruitment and retention of study participants. The inclusion of community members as stakeholders would enable the community to make informed decisions about their participation in the research while learning about its potential impact, and to also serve as a foundation for active community participation in the dissemination of study results.
Program managers, officers, and directors in non-profit funding organizations can create seed funding opportunities for CBOs that help develop programs to support quality research and outcomes. Programs can create and provide tools and resources for researchers, communities, and other stakeholders to inform the populations they serve. Program officers, managers and directors can create programmatic opportunities that will inform researchers on best practices for engaging with communities of interest. Seed funding is another mechanism for creating resources such as community health programs, development of educational materials, and tutorials on how to complete CITI trainings.

It is the responsibility of the researcher to ensure that the community is properly informed and that research teams have the social skills necessary to interact with study participants. It would be advantageous for researchers to engage with aging PLWH to learn about their lived experiences and to establish trust. High-quality, ethically responsible research should serve a purpose to the researcher and benefit the community's health. Research conducted without consideration for the community can be detrimental and tarnish the reputation of researchers. Engaging with community leaders is vital to producing information and data that can be used in an impactful way. Researchers should consider including the community of interest in the development of study protocols and plan for the dissemination of study findings to ensure the impact of the research.

Researchers should prioritize informing potential study volunteers and participants about research opportunities, and CBOs could also establish a code of conduct for researchers and participants. Community leaders can create community navigation guides for researchers to better prepare them to enter a community of interest. Community advisory boards can also contribute by informing volunteers on how to partner with researchers and interpret research protocols, and by translating study materials (informed consent, study documents, etc.) to wording that is relevant and comprehensive to the community (Cox et al., 1998; Isler et al., 2015; King et al., 2014; Quinn, 2004; Strauss et al., 2001; Zhao et al., 2019).

\section{Discussion}

\section{Bringing the Perspectives Together: Common Themes}

Each research stakeholders' individual perspective identified some ethical shortcomings that stem from research practices illustrated by this case, demonstrating best practices for researchers, as well as the value of community involvement in research. It also highlights the difference between being an ethical researcher, and following the minimum standards set out in the regulations (Department of Health, Education, and Welfare, 2014; U.S. Department of 
Health and Human Services, 2017; World Medical Association, 2013). Seldom are true situations from the field brought into the academic literature, and even more seldom are these situations reflected upon by multiple stakeholders in the research enterprise. This represents the first case study that we know of with this framing and analysis. Realistic examples such as this can provide context and a shared learning experience for community members, funders, ethicists, IRBs, and investigators who may be planning future community partnered activities. Several interrelated themes are present across each perspective: community members as experts and partners; research should benefit the community; and clear communication is key. These are each outlined briefly below.

Community members as experts and partners. Each commentator regarded communities as vital resources in the research process due to their lived experiences, which gives them unique knowledge and expertise. When communities' expertise is leveraged by investigators, the result is higher quality, more useful data, and increased opportunities for creating real-world impact. Investigators can use community members' expertise throughout the research process, but it can be particularly helpful to do so early on when developing study materials, such as surveys, interview guides, recruitment methods, and IRB protocols. The commentators also assert that communities should be respected and treated as valuable resources for ethical research, which may include compensating them for their time and efforts.

Research should benefit the community. The commentators also discussed the importance of research benefiting the community. For example, research should serve the needs of the community, rather than the needs of the researcher. At the very least, benefits should include sharing findings and data with the community, which communities can use to inform programs and interventions and advance their knowledge (Day et al., 2018; MacQueen et al., 2012). Benefits can also include increasing capacity by providing community members with training necessary for the conduct of future research (Brown \& Sugarman, 2020). Ensuring that research benefits the community helps avoid exploitative relationships between researchers and communities and helps ensure that communities have a vested interest in contributing to the research.

Clear communication is key. Finally, each commentator expressed the importance of having clear communication between researchers and community members. This communication is particularly important for establishing a shared understanding of expectations and roles of researchers and community members. Communication is also important for aligning the research with the study priorities of the community. Clear communication throughout the research process shows respect and helps build trust, both of which establish a foundation for future research opportunities and collaborations.

These themes and the commentators' perspectives more broadly reflect aspects of Community-Based Participatory Research, Good Participatory Practices, and the Denver Principles, and illustrate how the case is antithetical to each of these frameworks, described below.

\section{CBPR, Good Participatory Practices, Denver Principles, CIOMS Guidelines}

Community-based participatory research. CBPR is a method of research in which community participation is enshrined in the research process. It centers on the promotion of colearning among researchers and community members, which helps to equalize power between research participants and researchers, transfers expertise between participants and researchers, and establishes shared ownership of the research and its outcomes for successful implementation and uptake (Mills et al., 2006; Rhodes et al., 2010; Wallerstein \& Duran, 2010;). In doing so, carrying out CBPR necessitates clear communication between researchers and community members, treating the community as a resource that enhances the research, and providing benefits to the community. In each of these aspects, the case exemplifies a manner of research that is thoroughly discordant with CBPR, possibly due to the fact that the survey research was not planned as CBPR in the mind of the researcher.

Good participatory practices. Community engagement and giving community members a voice in the research process is at the heart of GPP. The specific GPP guidelines developed by UNAIDS and AVAC provide the steps necessary for ensuring that community members are included and heard throughout the research process (UNAIDS/AVAC, 2011). Core principles of GPP include respect, mutual understanding, transparency, and community autonomy. As the commentators suggest, GPP were clearly not utilized in the case. On the contrary, Dr. JM demonstrated a lack of transparency and disrespect, which prevented mutual understanding and curtailed the community's autonomy. While the GPP were created specifically for biomedical HIV research, the core principles can be applied to all research including survey research.

Denver principles. What first started out as a manifesto, the Denver Principles which were written during the 1983 Annual Gay and Lesbian Health Conference in Denver, Colorado represented a self-empowerment movement that became a lifeline for people with HIV around the world. At the core of the Denver Principles is treating people as "whole people," deserving of full respect, autonomy, and inclusion ("The Denver Principles," 1983). Considering 
communities as resources and treating them as such, ensuring that research benefits the community, and clearly communicating with community members throughout the research process are all consistent with the foundational aspects of the Denver Principles.

CIOMS guidelines. In contrast to the GPP, which were created with biomedical HIV research in mind, the CIOMS international ethical guidelines for health-related research involving humans is meant for all health research including survey research (CIOMS, 2016). With 25 guidelines in total, this case study falls short of guideline 7 , which provides specific recommendations for engaging with the community from which research participants will be drawn. Guideline 8 also points to the creation of a memorandum of understanding to promote inclusion, mutual understanding, and social justice.

\section{A Way Forward}

We argue that this case study illustrates an example of mismatched expectations and lack of collaboration on the part of the researcher, which is specifically what CBPR is meant to address (Rhodes et al., 2010; Wallerstein \& Duran, 2010). It also illustrates the perceived unequal power dynamics between researchers and community members.

As a result, local community members agreed that the HIV researcher neither adhered to GPP nor to the recommended practices from the CIOMS guidelines (CIOMS, 2016). The community members took this interaction as a learning experience and created both a collaboration request form for non-clinical research (Figure 1) and a user-friendly GPP checklist (Figure 2), which will be sent to investigators in advance of their potential collaboration with the local community. In addition, while no MOU was prepared for this research to help set expectations, the community is now well informed to utilize an MOU in future research. This can be built from the Stakeholder Engagement Toolkit chapter on "Secure Commitment to Guiding Principles" which includes obtaining written commitment to principles by key members of the research team (MacQueen et al., 2012).

This study is not without its limitations. The case study respondents were all selected by the corresponding author due to their stakeholder role. Therefore they may have been more biased towards CBPR and viewing community members as research partners rather than research subjects, as well as judging researcher conduct using a CBPR lens. In addition, since only four respondents were chosen (one per stakeholder group), the responses of respondents are not generalizable to their more global stakeholder group. Finally, we did not conduct formal qualitative analysis of key topics that emerged in the short commentaries, but respondents did work together to reach consensus on key themes.
It is our hope that Figures 1 and 2, created in collaboration with stakeholders in the research process will significantly reduce or greatly limit the unethical conduct of research in the future. We also hope that others can learn from our experience and this paper can be used as a tool to help researchers, ethicists, IRB members, funders, students, and most importantly, community members, properly and ethically navigate the research process with study participants and community members.

\section{Best Practices}

To the extent possible, researchers should educate themselves on the proper practice of community-engaged research. In the HIV field, this can include availing themselves with deliberative methods, GPP, HPTN Ethics Trials Guidelines for Research, the Denver Principles, and the standards for engagement in CIOMS which applies to all health research (Brown \& Sugarman, 2020; CIOMS, 2016; Emanuel et al., 2004, 2008). Towards this end, researchers can utilize our real-life case study example as a tool for understanding the impact and consequences of negative researcher behavior on communities. Our easy-to-use GPP checklist and collaboration form can be used and modified as a starting point for discussions between community members and researchers to clarify expectations and increase the likelihood that community-based research is conducted ethically and respectfully.

\section{Research Agenda}

More case studies, whether actual or hypothetical, are needed, particularly when it comes to community-engaged research. Our recommendation to other investigators who hope to contribute such case studies is to be aware that few community members may be willing to bring issues such as these to the forefront, particularly if the research will bring resources and other funding to the community despite any neglect by researchers of recognized GPP/and stakeholder engagement protocols.

\section{Educational Implications}

This case study may in itself be an educational resource for those who will be actively participating in some way in the research process. It was written to reflect multiple perspectives on a situation that occurred in HIV research. Possible audiences include stakeholders such as researchers, community members, IRB members and funders to better understand optimal and suboptimal interaction with potential study communities and participants in research projects. In addition, the case can be seen as an argument for better uptake of existing educational resources on GPP (e.g., 


\section{Requester}

\begin{tabular}{|c|c|c|c|c|c|}
\hline \multicolumn{6}{|l|}{ Date of request: } \\
\hline Please write the & \multicolumn{3}{|c|}{ Individuals Name and Title } & \multicolumn{2}{|c|}{ Organization Name } \\
\hline $\begin{array}{c}\text { Type of Request } \\
\text { (circle all that apply): }\end{array}$ & Data & $\begin{array}{l}\text { Access to } \\
\text { Patients }\end{array}$ & $\begin{array}{l}\text { Letter of } \\
\text { Support }\end{array}$ & $\begin{array}{l}\text { Conference/ } \\
\text { Meeting }\end{array}$ & $\begin{array}{l}\text { Other } \\
\text { specify }\end{array}$ \\
\hline \multicolumn{6}{|l|}{$\begin{array}{l}\text { Please tell us more } \\
\text { about your request } \\
\text { and the purpose: }\end{array}$} \\
\hline \multicolumn{6}{|l|}{$\begin{array}{l}\text { Describe our role } \\
\text { be regarding your } \\
\text { request: }\end{array}$} \\
\hline \multicolumn{6}{|l|}{$\begin{array}{c}\text { What is your } \\
\text { timeline? }\end{array}$} \\
\hline $\begin{array}{l}\text { Please tell us about } \\
\text { the benefit of this } \\
\text { collaboration to us. } \\
\text { Examples are } \\
\text { funding, salary } \\
\text { support, publication } \\
\text { authorship, } \\
\text { conference } \\
\text { registration, etc. }\end{array}$ & & & & & \\
\hline
\end{tabular}

Requestee (the following should be filled out by the organization receiving the request)

\begin{tabular}{|c|c|c|}
\hline Approval: & Yes (list why) & No (list why) \\
\hline Signature and Date: & & \\
& & \\
\hline
\end{tabular}

Notes (optional):

Figure I. Collaboration request template. 


\section{Before Research:}

1. Formative research activities

Develop a well-planned research activity

- Propose it to research team and the to stakeholders to assure that everyone is on board and believes it is a research activity worth conducting/funding

2. Stakeholder advisory mechanisms

U Use mechanisms to create meaningful dialogue with participants in order to create more quality results

3. Stakeholder engagement plan

- Researchers should:

- Keep in mind the sensitive content of HIV and present to research subjects in an appropriate matter

- Keep in mind the guiding principles of GPP in biomedical HIV prevention trials/ the area of research: respect, mutual understanding, integrity, transparency, accountability, \& community stakeholder autonomy

4. Stakeholder education plan

To provide relevant education about a specific planned trial — and about HIV biomedical research in general — in order to enhance research literacy

5. Communication plan

Research teams should:

- Be involved communication networks to avoid any management/ communication issues

- Talk to stakeholders before experimental trials to ensure that designs and procedures are effective for everyone

6. Issues management plan

- Have a systematic plan that you will use to solve problems

7. Site selection

To select a site to be funded for trial protocol, inclusion in a multisite trial or a trial network

8. Protocol development

a Protocol Development $=$ the process of generating a trial protocol so that it is of high caliber. Once all trials are completed, all data must be distributed to everyone. This allows transparency that will hopefully build trust.

\section{During Research:}

9. Informed consent process

T To provide a competent individual with enough information about a trial to make an independent decision whether or not to participate in the trial

10. Standard of HIV preventions

Negotiate HIV prevention package. What should and will the patients receive? Identify any possible problems

11. Access to HIV care and treatment

Participants who obtain HIV during the trial must have access to HIV care and treatment

12. Non HIV-related care

T Trials should have access to non related HIV related care

13. Policies on trial-related harms

Discuss what will happen if participants experience harm during trials. Including social harm. List all possible harms. Policies should be made from this

14. Trial accrual, follow-up, and exit

- Design socially and culturally acceptable strategies for recruitment, screening, enrollment, follow up and exit

\section{Post Trial Research:}

15. Trial closure and results dissemination

Assure proper dissemination of trial results

16. Post-trial access to trial products or procedure

T The product or procedure that is tested should be available to those who participated in the project

Figure 2. Good Participatory Practices checklist. 
https://engage.avac.org/courses/gpp-online-training-_aprjun-2018/).

\section{Acknowledgments}

The authors would like to thank the community members who participated in the research described in this case study. Thanks to Jo Gerrard for editorial assistance.

\section{Declaration of Conflicting Interests}

The author(s) declared no potential conflicts of interest with respect to the research, authorship, and/or publication of this article.

\section{Funding}

The author(s) received no financial support for the research, authorship, and/or publication of this article.

\section{References}

Adhikari, B., Pell, C., \& Cheah, P. Y. (2019). Community engagement and ethical global health research. Global Bioethics, 31(1), 1-12.

AVAC. (2018). Good participatory practice online training. https://engage.avac.org/courses/gpp-online-training-_aprjun-2018/

Barrett, N. J., Rodriguez, E. M., Iachan, R., Hyslop, T., Ingraham, K. L., Le, G. M, Martin, K., Haring, R. C., Rivadeneira, N. A., Erwin, D. O., Fish, L. J., Middleton, D., Hiatt, R. A., Patierno, S. R., Sarkar, U., \& Gage-Bouchard, E. A. (2020). Factors associated with biomedical research participation within community-based samples across 3 National Cancer Institutedesignated cancer centers. Cancer, 126(5), 1077-1089.

Bastida, E. M., Tseng, T.-S., McKeever, C., \& Jack, L. (2010). Ethics and community-based participatory research: Perspectives from the field. Ethics in Health Education, 11(1), 16-20.

Beecher, H. K. (1966). Consent in clinical experimentation: Myth and reality. JAMA, 195(1), 34-35. https://www.ncbi.nlm.nih. gov/pubmed/5951827

Brandt, A. M. (1978). Racism and research: The case of the Tuskegee Syphilis study. Hastings Cent Rep, 8(6), 21-29. https://www.ncbi.nlm.nih.gov/pubmed/721302

Brown, B., \& Sugarman, J., for the HPTN Ethics Working Group. (2020). Why ethics guidance needs to be updated for contemporary HIV prevention research. Journal of the International AIDS Society, 23(5), e25500. https://doi. org.10.1002/jia2.25500

Browne, J. L., Rees, C. O., van Delden, J. J. M., Agyepong, I., Grobbee, D. E., Edwin, A., Klipstein-Grobusch, K., \& van der Graaf, R. (2019). The willingness to participate in biomedical research involving human beings in low- and middleincome countries: A systematic review. Tropical Medicine \& International Health, 24(3), 264-279.

Council for International Organizations of Medical Sciences (CIOMS). (2016). International ethical guidelines for healthrelated research involving humans ( 2 nd ed.). CIOMS.

Cox, L. E., Rouff, J. R., Svendsen, K. H., Markowitz, M., \& Abrams, D. I. (1998). Community advisory boards: Their role in AIDS clinical trials. Terry Beirn Community Programs for Clinical Research on AIDS. Health and Social Work, 23(4), 290-297.

CTSA. (2011). Clinical and translational science awards consortium. Community engagement key function committee task force on the principles of community engagement. https:// www.atsdr.cdc.gov/communityengagement/

Day, S., Blumberg, M., Vu, T., Zhao, Y., Rennie, S., \& Tucker, J. D. (2018). Stakeholder engagement to inform HIV clinical trials: A systematic review of the evidence. Journal of the International AIDS Society, 21(Suppl. 7), e25174.

Department of Health, Education, and Welfare; National Commission for the Protection of Human Subjects of Biomedical and Behavioral Research. (2014). The Belmont Report. Ethical principles and guidelines for the protection of human subjects of research. https://www.hhs.gov/ohrp/sites/ default/files/the-belmont-report-508c FINAL.pdf

Emanuel, E. J., Grady, C., Crouch, R. A., Lie, R. K., Miller, F. G., \& Wendler, D. (2008). The Oxford textbook of clinical research ethics. Oxford University Press.

Emanuel, E. J., Wendler, D., Killen, J., \& Grady, C. (2004). What makes clinical research in developing countries ethical? The benchmarks of ethical research. The Journal of Infectious Diseases, 189(5), 930-937. https://doi.org/10.1086/381709

Haggerty, K. D. (2004). Ethics creep: Governing social science research in the name of ethics. Qualitative Sociology, 27(4), 391-414.

HIV/AIDS Network Coordination (HANC). (2014). Recommendations for community engagement in HIV/AIDS research. https://www.hanc.info/cp/resources/Documents/Recommendations \%202014\%20FINAL\%206-5-14\%20rc.pdf

Isler, M. R., Miles, M. S., Banks, B., Perreras, L., Muhammad, M., Parker, D., \& Corbie-Smith, G. (2015). Across the miles: Process and impacts of collaboration with a rural community advisory board in HIV research. Progress in Community Health Partnerships: Research, Education, and Action, 9(1), 41-48.

King, K. F., Kolopach, P., Merritt, M. W., \& Lavery, J. L. (2014). Community engagement and the human infrastructure of global health research. BMC Medical Ethics, 15(84), 1-6.

Klitzman, K. (2013). How IRBs view and make decisions about cocercion and undue influence. Journal of Medical Ethics, 39(4), 224-229.

MacQueen, K. M., Harlan, S. V., Slevin, K. W., Hannah, S., Bass, E., \& Moffett, J. (2012). Stakeholder engagement toolkit for HIV prevention trials. https://www.fhi360.org/sites/default/ files/media/documents/Stakeholder_Engagement_ToolkitFULL_0.pdf

Milgram, S. (1963). Behavioral study of obedience. The Journal of Abnormal and Social Psychology, 67, 371-378. https://doi. org $/ 10.1037 / \mathrm{h} 0040525$

Mills, E. J., Nachega, J. B., Bangsberg, D. R., Singh, S., Rachlis, B., Wu, P., Wilson, K., Buchan, I., Gill, C. J., \& Cooper, C. (2006). Adherence to HAART: A systematic review of developed and developing nation patient-reported barriers and facilitators. PLoS Medicine, 3(11), e438.

Partner, P. (n.d.). Patient involvement in clinical research: A guide for patient organisations and patient representatives. https:// 
www.geneticalliance.org.uk/media/1602/patientspartnerforpatientorgs.pdf

Quinn, S. C. (2004). Protecting human subjects: The role of community advisory boards. American Journal of Public Health, 94(6), 918-922.

Rhodes, S. D., Malow, R. M., \& Jolly, C. (2010). Communitybased participatory research: A new and not-so-new approach to HIV/AIDS prevention, care, and treatment. AIDS Education and Prevention, 22(3), 173-183. https://doi.org/10.1521/ aeap.2010.22.3.173

Shippee, N. D., Domecq Garces, J. P., Prutsky Lopez, G. J., Wang, Z., Elraiyah, T. A., Nabhan, M., Brito, J. P, Boehmer, K., Hasan, R., Firwana, B., Erwin, P. J., Montori, V. M., \& Murad, M. H. (2015). Patient and service user engagement in research: A systematic review and synthesized framework. Health Expect, 18(5), 1151-1166. https://doi.org/10.1111/ hex. 12090

Smith, J. (2018). Parasitic and parachute research in global health. Lancet Global Health, 6(8), E838. https://doi.org/10.1016/ S2214-109X(18)30315-2

Strauss, R. P., Sengupta, S., Quinn, S. C., Goeppinger, J., Spaulding, C., Kegeles, S. G., \& Millett, G. (2001). The role of community advisory boards: Involving communities in the informed consent process. American Journal of Public Health, 91(12), 1938-1943.

Subica, A., \& Brown, B. (2020). Addressing health disparities through deliberative methods: Citizen's panels for health equity. American Journal of Public Health, 110(2), 166-173. https://doi.org/10.2105/AJPH.2019.305450

The Denver Principles. (1983). Geneva. UNAIDS. http://data. unaids.org/pub/externaldocument/2007/gipa1983denverprinciples_en.pdf

U.S. Department of Health and Human Services. (2017). Code of federal regulations - Title 45 Public Welfare CFR 46. https:// www.ecfr.gov/cgi-bin/retrieveECFR?gp $=\& \mathrm{SID}=83 \mathrm{~cd} 09 \mathrm{e} 1 \mathrm{c} 0$ f5c6937cd9d7513160fc 3 f \&pitd $=20180719 \& n=p t 45.1 .46 \& r$ =PART\&ty=HTML, https://www.hhs.gov/ohrp/sites/default/ files/ohrp/policy/ohrpregulations.pdf

UNAIDS/AVAC. (2011). Good participatory practice: Guidelines for biomedical HIV prevention trials. Geneva. UNAIDS. https:// www.avac.org/sites/default/files/resource-files/Good $\% 20$ Participatory\%20Practice\%20guidelines_June_2011.pdf

Wallerstein, N., \& Duran, B. (2010). Community-based participatory research contributions to intervention research: The intersection of science and practice to improve health equity. American Journal of Public Health, 100(Suppl. 1), S40-S46. https://doi.org/10.2105/AJPH.2009.184036

Wallerstein, N., Duran, B., Oetzel, J. G., \& Minkler, M. (2018). Community-based participatory research for health. JosseyBass.

WorldMedicalAssociation.(2013).DeclarationofHelsinki:Ethical principles for medical research involving human subjects. https://www.wma.net/policies-post/wma-declaration-of- helsinki-ethical-principles-for-medical-research-involvinghuman-subjects/

Zhao, Y., Fitzpatrick, T., Wan, B, Day, S., Mathews, A., \& Tucker, J. D. (2019). Forming and implementing community advisory boards in low- and middle-income countries: A scoping review. BMC Medical Ethics, 20(73), 1-11.

\section{Author Biographies}

Brandon Brown is an associate professor of medicine at the University of California, Riverside. With over 130 publications, his research interests include local and global HIV prevention and treatment, as well as ethical issues related to payment in research. He led the development of the case study and solicited the stakeholder perspectives.

Jeff Taylor has been HIV+ for 35 years and has served as a community advisor to many research groups. He currently serves on the National Cancer Institute's AIDS Malignancy Consortium's ANCHOR Study CAB; the community advisory board for the CARE Collaboratory, the Gladstone amfAR Cure Institute CAB, the UC Riverside Clinical IRB, and on the Clinical Advisory Panel for CIRM-funded trial at City of Hope. He is the director of the HIV \& Aging Research Project-Palm Springs, was involved with the case study presented in this paper, and contributed his perspective on the case study.

Karine Dubé is an assistant professor in the Public Health Leadership Program at the University of North Carolina Gillings School of Global Public Health. Her current work focuses on integrating socio-behavioral sciences, biomedical research, ethics, patient and community and patient engagement in infectious diseases research. She contributed her perspective on the case study.

Dario Kuzmanović is the assistant vice chancellor at the University of California, Riverside and a member of the Joint Centre for Bioethics at University of Toronto. He is a research ethicists with who serves on public and private boards and has consulted for numerous organizations and groups - including the World Health Organization. He contributed his perspective on the case study.

Yasmeen Long is a Director at FasterCures, a center of the Milken Institute, and a public health and research professional who focuses on health outcomes, policy, patient and stakeholder engagement, and global health. Her work focuses on eliminating disparities, promoting health equity, and improving quality of life for underrepresented and vulnerable communities. She contributed her perspective on the case study.

Logan Marg is a user experience researcher and frequent collaborator with the lead author. He has contributed to numerous community-engaged research projects. For the present paper, he synthesized and analyzed the stakeholders' perspectives, assisted with manuscript writing, and prepared the manuscript. 